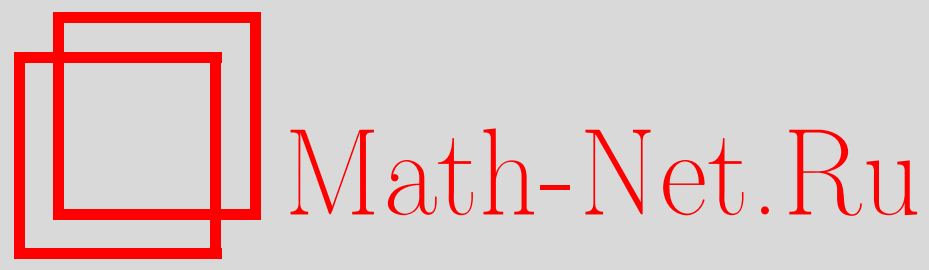

Б. З. Шапиро, Нормальные формы зонтика Уитни относительно контактной группы, сохраняющей конус, Функи. анализ и его прил., 1997, том 31, выпуск 2, 91-94

DOI: https://doi.org/10.4213/faa469

Использование Общероссийского математического портала MathNet.Ru подразумевает, что вы прочитали и согласны с пользовательским соглашением

http://www . mathnet.ru/rus/agreement

Параметры загрузки:

IP : 3.81 .55 .215

26 апреля 2023 г., 14:33:36

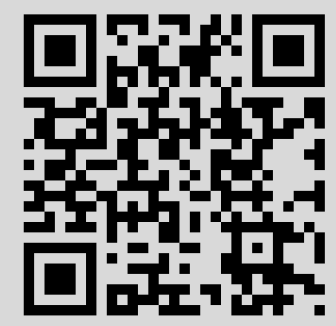




\title{
Нормальные формы зонтика Уитни относительно контактной группы, сохраняющей конус
}

\author{
(c) 1997. Б. З. ШАПиРО
}

1. Результаты. Пусть $\mathscr{S} \subset \mathbb{R}^{n}$ - стратифицированное подмногообразие, содержащее начало координат. Обозначим через $\mathscr{K}$ контактную группу, т.е. элементы группы $\mathscr{K}$ - это диффеоморфизмы произведения $\left(\mathbb{R}^{l} \times \mathbb{R}^{n}, 0\right)$, сохраняющие (а) проекцию на $\mathbb{R}^{l}$ (индуцирующие диффеоморфизм пространства $\left.\mathbb{R}^{l}\right)$ и (в) подпространство $\left(\mathbb{R}^{l} \times 0\right)$, см. [2]. Назовем два ростка отображений $f_{1}, f_{2}:\left(\mathbb{R}^{l}, 0\right) \rightarrow\left(\mathbb{R}^{n}, 0\right) \quad \mathscr{K}$-эквивалентными, если существует такой диффеоморфизм пространства $\left(\mathbb{R}^{l}, 0\right)$ и росток отображения $M:\left(\mathbb{R}^{l}, 0\right) \rightarrow G L\left(\mathbb{R}^{n}\right)$, что $f_{1}(x)=M(x) f_{2}(g(x))$. Наконец, пусть $\mathscr{K}_{\mathscr{S}}$ обозначает подгруппу в $\mathscr{K}$, состоящую из диффеоморфизмов $h \in \mathscr{K}$, таких, что $h\left(\mathbb{R}^{l} \times \mathscr{S}\right) \subset \mathbb{R}^{l} \times \mathscr{S}$.

Цель этой заметки - классифицировать ростки отображений $f:\left(\mathbb{R}^{2}, 0\right) \rightarrow$ $\left(\mathbb{R}^{3}, 0\right)$ относительной контактной группы $\mathscr{K}_{\mathscr{C}}$, где $\mathscr{C}$ - обычный конус с вершиной в нуле, и мы дополнительно предполагаем, что образ отображения $f$ зонтик Уитни. Заметим, что нормальные формы взаимного расположения зонтика Уитни и гладкого ростка гиперповерхности в $\mathbb{R}^{3}$ относительно право-левой и контактной эквивалентности изучались в работе [4]. Важные общие свойства контактной группы $\mathscr{K}_{\mathscr{S}}$, где $\mathscr{S}$ - произвольное стратифицированное подмногообразие, рассмотрены в [3].

Напомним, что любой зонтик Уитни $f:\left(\mathbb{R}^{2}, 0\right) \rightarrow\left(\mathbb{R}^{3}, 0\right)$ определяет полный флаг $F l$ в касательном пространстве к $\mathbb{R}^{3}$ в начале координат, см. рис. 1 и [1]. Прямая $l$ этого флага является касательным пространством к образу $f$ в 0 , а плоскость $L$ флага (взятая с кратностью 2) совпадает с касательным конусом к зонтику в 0 . Назовем односвязные компоненты дополнения к фиксированному квадратичному конусу $\mathscr{C} \subset \mathbb{R}^{3}$ гиперболическими, а неодносвязную компоненту — негиперболической.

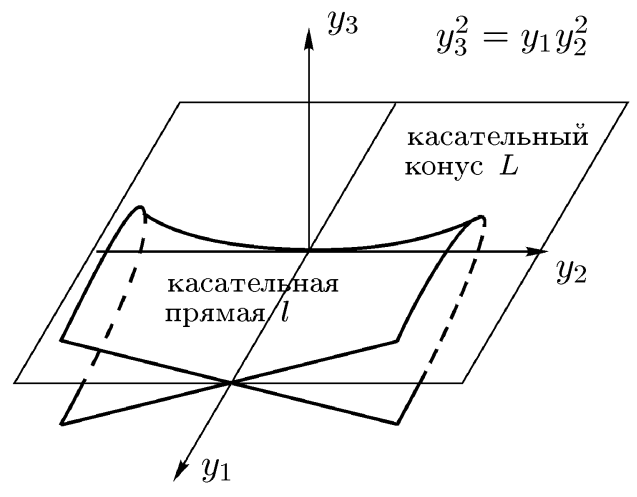

Рис. 1. Стандартный зонтик Уитни

ПРеДЛОЖЕнИЕ. Существует шесть орбит действия группы $G L_{3}$ на множестве пар $(F l, \mathscr{C})$ :

1) прямая l проходит через гиперболические компоненты $\mathbb{R}^{3} \backslash \mathscr{C}$ (общее положение);

2) прямая $l$ проходит через негиперболическую компоненту и плоскость $L$ пересекает $\mathscr{C}$ по паре прямьх (общее положение);

3) прямал $l$ лежит в негиперболической компоненте и плоскость $L$ не пересекает $\mathscr{C}$ вне 0 (общее положение); 
4) прямая $l$ лежит в негиперболической компоненте и $L$ касается $\mathscr{C}$ $(\operatorname{codim}=1)$

5) прямая $l$ лежит на $\mathscr{C}$ и $L$ пересекает $\mathscr{C}$ по паре прямых, одна из которых совпадает с $l(\operatorname{codim}=1)$;

6) прямая $l$ лежит на $\mathscr{C}$ и $L$ касается $\mathscr{C}$ вдоль $l(\operatorname{codim}=2)$.

Теперь мы можем привести список нормальных форм ростков отображений $f$ относительно контактной группы $\mathscr{K}_{\mathscr{C}}$, где $\mathscr{C}$ - квадратичный конус, заданный уравнением $Q: y_{1} y_{2}-y_{3}^{2}=0$.

Основная теорема. Росток отображения $f:\left(\mathbb{R}^{2}, 0\right) \rightarrow\left(\mathbb{R}^{3}, 0\right)$, образ которого является зонтиком Уитни и такой, что $f^{*} Q$ имеет изолированную особенность в нуле, приводится группой $\mathscr{K}_{\mathscr{C}} \kappa$ одной из следующих нормальных форм в соответствии с классификачией пар $(F l, \mathscr{C})$. (Напомним, что $Q=y_{1} y_{2}-y_{3}^{2}$ задает конус $\mathscr{C}$.)

1) $f: y_{1}=x_{1}, y_{2}=x_{1}+x_{2}^{2}, y_{3}=0$ (здесь $\left.f^{*} Q \simeq A_{3}^{-}\right)$;

2) $f: y_{1}=x_{1}, y_{2}=-x_{1}+x_{2}^{2}, y_{3}=0$ (здесь $\left.f^{*} Q \simeq A_{3}^{-}\right)$;

3) $f: y_{1}=x_{1}, y_{2}=-x_{1}, y_{3}=x_{2}^{2}\left(\right.$ здесь $\left.f^{*} Q \simeq A_{3}^{+}\right)$;

4) $f_{k}: y_{1}=x_{1}, y_{2}=-x_{1}+2 x_{2}^{2} \mp x_{2}^{k-1}, y_{3}=x_{2}^{2}, k \geqslant 4$ (здесь $\left.f_{k}^{*} Q \simeq A_{k}^{ \pm}\right)$;

5) $f_{k}: y_{1}=x_{1}, y_{2}=x_{2}^{2} \pm x_{1}^{k-2}, y_{3}=0, k \geqslant 4\left(\right.$ здесь $\left.f^{*} Q \simeq D_{k}^{ \pm}\right)$;

6) $f_{5}: y_{1}=x_{1}, y_{2}=x_{1} x_{2}, y_{3}=x_{2}^{2}\left(\right.$ здесь $\left.f^{*} Q \simeq D_{5}\right)$.

Обратите внимание, что во всех случаях, кроме 6), образом 2-струи отображения $f$ является не зонтик Уитни, а более простая гиперповерхность с неизолированной особенностью. Это проявление того обстоятельства, что $\mathscr{K}_{\mathscr{C}}$-Эквивалентность является довольно грубой и не сохраняет даже стабильного типа отображения $f$. (В приведенной выше классификации в скобках указан тип ростка функции $f^{*} Q$ в соответствии со стандартной классификацией особенностей ростков функций, см. [1].)

Ниже приведена диаграмма примыканий особенностей отображения $f$. (Жирные цифры на рис. 2 соответствуют одному из шести случаев основной теоремы.)

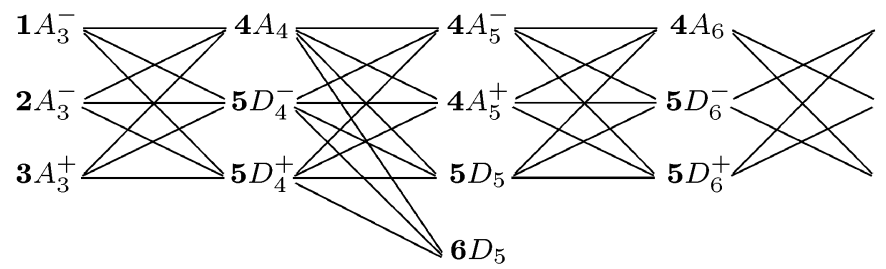

\section{Рис. 2. Примыкания орбит $\mathscr{K}_{\mathscr{C}}$-действия}

Эта заметка продолжает классификацию ростков гладких иммерсий в $\mathbb{R}^{n}$ относительно группы $\mathscr{K}_{\mathscr{C}}$, начатую в [5]. Здесь изучен первый нетривиальный иррегулярный случай, т.е. случай когда образ отображения $f$ особый. Автор благодарен В. Горюнову за ряд полезных обсуждений и упрощений первоначальных вычислений.

2. Доказательства. Начнем доказательство теоремы со следующего замечания: 1 -струя отображения $f:\left(\mathbb{R}^{2}, 0\right) \rightarrow\left(\mathbb{R}^{3}, 0\right)$ ранга 1 приводится действием группы $\mathscr{K}_{\mathscr{C}}$, где конус $\mathscr{C}$ задан уравнением $y_{1} y_{2}-y_{3}^{2}=0$, к одному из трех видов (a) $\left(x_{1}, x_{1}, 0\right)$, (b) $\left(x_{1},-x_{1}, 0\right)$ или (c) $\left(x_{1}, 0,0\right)$. 
Действительно, прямая, проходящая через 0, приводится к одному из этих трех видов линейной группой, сохраняющей $\mathscr{C}$.

Лемма. (А) 2-струя любого отображения $f:\left(\mathbb{R}^{2}, 0\right) \rightarrow\left(\mathbb{R}^{3}, 0\right)$ ранга 1 приводится действием $\mathscr{K}_{\mathscr{C}} \kappa$ одной из следуюших форм: 1) $\left(x_{1}, x_{1}+x_{2}^{2}, 0\right)$; 2) $\left(x_{1},-x_{1}+x_{2}^{2}, 0\right)$; 3) $\left(x_{1},-x_{1}, x_{2}^{2}\right)$; 4) $\left.\left(x_{1},-x_{1}+2 x_{2}^{2}, x_{2}^{2}\right) ; 5\right)\left(x_{1}, x_{2}^{2} \pm x_{1}^{2}, 0\right)$; $\left.\left.\left.\left.\left.5^{\prime}\right)\left(x_{1}, x_{2}^{2}, 0\right) ; 6\right)\left(x_{1}, x_{1} x_{2}, x_{2}^{2}\right) ; 7\right)\left(x_{1}, x_{1}^{2}, x_{2}^{2}\right) ; 8\right)\left(x_{1}, 0, x_{2}^{2}\right) ; 9\right)\left(x_{1}, x_{1}^{2}, 0\right)$; 10) $\left(x_{1}, x_{1}, 0\right)$; 11) $\left.\left(x_{1},-x_{1}, 0\right) ; 12\right)\left(x_{1}, 0,0\right)$.

(В) Последние шесть из приведенных форм не могут возникнуть, если образ $f$ - зонтик Уитни.

Доказательства этой леммы и теоремы основаны на стандартной технике приведения к нормальной форме, описанной в [1] и для нашего частного случая в [5]. Основная идея - рассмотреть $\mathscr{O}_{x}$-модуль $\mathfrak{M}_{\mathscr{C}}(f)$ векторных полей, касательных к орбите $\mathscr{K}_{\mathscr{C}}(f)$ в точке $f$, и вычислить младшие струи некоторого базиса модуля $\mathfrak{M}_{\mathscr{C}}(f)$, зависящие от соответствующих младших струй отображения $f$. Согласно [5], алгебра Ли группы $\mathscr{K}_{\mathscr{C}}$ имеет базис, состоящий из шести векторных полей, а именно $\partial / \partial x_{1}, \partial / \partial x_{2}$ и еще четырех полей, касательных к $\mathscr{C}$. Одно из этих полей - это эйлерово поле $\left(y_{1} \partial / \partial y_{1}, y_{2} \partial / \partial y_{2}, y_{3} \partial / \partial y_{3}\right)$, а остальные три - эти 2-миноры матрищы $\left(\begin{array}{ccc}\partial / \partial y_{1} & \partial / \partial y_{2} & \partial / \partial y_{3} \\ Q_{y_{1}} & Q_{y_{2}} & Q_{y_{3}}\end{array}\right)$, где $Q=y_{1} y_{2}-y_{3}^{2}$ и $Q_{y_{i}}=\partial Q / \partial y_{i}$. Чтобы получить базис модуля $\mathfrak{M}_{\mathscr{C}}(f)$, надо применить указанные шесть полей к $f$.

НАБРОСОК ДОКАЗАТЕЛЬСТВА ЛЕМмЫ. Часть (А). ВЫписывая векторные поля, можно непосредственно увидеть, что если 1-струя отображения $f$ имеет тип (a) или (b), то можно устранить все члены разложения отображения $f$ степени $>1$, которые делятся на $x_{1}$. Кроме того, можно считать, что координата $y_{1}$ равна $x_{1}$. Следовательно, для 1-струй типа (a) и (b) достаточно рассмотреть 2-струи вида $\left(x_{1}, \pm x_{1}+\alpha x_{2}^{2}, \beta x_{2}^{2}\right)$.

Если $\beta=0$ и $\alpha \neq 0$, то можно произвести нормировку, положив $\alpha=1$, и получить нормальные формы 1) и 2). (Обратите внимание, что 2-струи $\left(x_{1}, x_{1}+\right.$ $\left.x_{2}^{2}, 0\right)$ и $\left(x_{1}, x_{1}-x_{2}^{2}, 0\right)$ 兓-эквивалентны, причем эквивалентность осуществляется при помощи линейного преобразования вида $\left(y_{1}^{\prime}=y_{2}, y_{2}^{\prime}=y_{1}, y_{3}^{\prime}=y_{3}\right)$.)

Если $\beta \neq 0$, то можно произвести нормировку, положив $\beta=1$. В этом случае после некоторых преобразований базиса полей получим для 1-струи отображения $f$ типа (b) касательное поле вида $\left(0,(\alpha-2) x_{2}^{2}, 0\right)$. Это означает, что при $\alpha \neq 2$ член $\alpha x_{2}^{2}$ координаты $y_{2}$ можно устранить, что приводит к нормальным формам 3) и 4). Наконец, случай $\alpha=\beta=0$ приводит к нормальным формам 9) и 10).

Рассмотрим теперь 1-струю отображения $f$ вида (c). В этом случае можно попрежнему устранить все члены, делящиеся на $x_{1}$ в координате $y_{3}$. Таким образом, достаточно рассмотреть 2-струи отображения $f$ вида $\left(x_{1}, \alpha x_{1}^{2}+\right.$ $\left.\beta x_{1} x_{2}+\gamma x_{2}^{2}, \delta x_{2}^{2}\right)$. Изучая базис векторных полей модуля $\mathfrak{M}_{\mathscr{C}}(f)$, получаем, что при $\gamma \neq 0$ можно устранить член $\delta x_{2}^{2}$ в координате $y_{3}$ и положить $\gamma=1$. Значит, достаточно рассмотреть 2-струю отображения $f$ вида $\left(x_{1}, \alpha x_{1}^{2}+\right.$ $\left.\beta x_{1} x_{2}+x_{2}^{2}, 0\right)$. Дополняя до полных квадратов и нормируя $\alpha$ в координате $y_{2}$, получаем нормальные формы 5$)$ и $5^{\prime}$ ).

Если $\gamma=0$ и $\delta \neq 0$, то можно положить $\delta=1$ и изучать 2-струю отображения $f$ вида $\left(x_{1}, \alpha x_{1}^{2}+\beta x_{1} x_{2}, x_{2}^{2}\right)$. Случай $\alpha \neq 0$ и $\beta=0$ приводит к 
нормальной форме 7). При $\alpha=\beta=0$ получаем 8). Если $\beta \neq 0$, то замена $\bar{x}_{2}=\beta x_{2}+\alpha x_{1}$ приводит к 2-струе вида $\left(x_{1}, x_{1} x_{2}, P\right)$, где $P$ - некоторый полином степени 2 . После устранения всех его членов, делящихся на $x_{1}$, получаем нормальную форму 6). Наконец, если все члены второго порядка равны нулю, то имеем 11).

Часть (В). Осталось показать, почему последние шесть нормальных форм не возникают, если образ отображения $f$ - зонтик Уитни. Действительно, касательный конус к зонтику в его вершине имеет вид $h_{L}^{2}=0$, где $h_{L}$ - линейная форма, задающая плоскость $L$ ассоциированного полного флага, см. рис. 1. Легко показать, что для любого $\phi$, лежащего в орбите $\mathscr{K}_{\mathscr{C}}(f)$, касательный конус к $\phi$ в вершине по-прежнему является квадратом линейной формы, если образ отображения $f$ - зонтик Уитни. Это условие не выполнено для нормальных форм 9)-12).

Предположим теперь, что образ отображения $f$ - зонтик Уитни и его 2-струя $\mathscr{K}_{\mathscr{C}}$-эквивалентна нормальным формам 7) или 8). Тогда ассоциированный флаг $F l=(l, L)$ имеет следующий вид: $l$ совпадает с осью $y_{1}$, a $L-$ с плоскостью $y_{2}=0$. Однако 2-струя любого отображения $f$, задающего зонтик Уитни и имеющего такой ассоциированный флаг, имеет вид $y_{1}=a_{1} x_{1}+a_{11} x_{1}^{2}$, $a_{1} \neq 0, y_{2}=b_{1} x_{1}+b_{11} x_{1}^{2}+b_{12} x_{1} x_{2}+b_{22} x_{2}^{2}, b_{12} \neq 0, y_{3}=c_{1} x_{1}+c_{11} x_{1}^{2}+c_{22} x_{2}^{2}$, $c_{22} \neq 0$. В процессе описанной выше редукции такая 2-струя приводится к виду $y_{1}=x_{1}, y_{2}=b_{11} x_{1}^{2}+b_{12} x_{1} x_{2}, b_{12} \neq 0, y_{3}=c_{22} x_{2}^{2}, c_{22} \neq 0$. Поскольку $b_{12} \neq 0$, мы получаем нормальную форму 6). Таким образом, нормальные формы 7) и 8) исключены из рассмотрения.

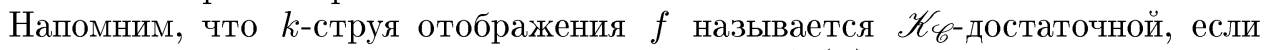
любое отображение вида $f+\varepsilon$ лежит в орбите $\mathscr{K}_{\mathscr{C}}(f)$ при условии, что $\varepsilon$ содержит только члены степени $\geqslant k+1$. (Подробности и критерий достаточности см. в [5] и [3].)

НАБРОСОК ДОКАЗАТЕЛЬСТВА ОСНОВНОЙ ТЕОРЕМЫ. 2-струя отображения $f$ является достаточной в случаях 1$), 2), 3)$ и 6$)$, поскольку ростки $f^{*} Q$, определенные этими 2-струями, являются простыми особенностями. В случае 4) достаточно рассмотреть только выписанную серию отображений $f_{k}$, поскольку можно устранить все члены, делящиеся на $x_{1}$, в координатах $y_{2}$ и $y_{3}$ и все члены вида $x_{2}^{l}, l \geqslant 3$, в координате $y_{3}$. Отображения $f_{k}$ попарно неэквивалентны. Наконец, для 2-струй вида 5) и $5^{\prime}$ ) можно устранить все члены, делящиеся на $x_{1}$, и все члены порядка $\geqslant 3$, делящиеся на $x_{2}$, в координате $y_{3}$. Это приводит в точности к указанным сериям, члены которых попарно неэквивалентны.

\section{ЛитеРАтУРА}

1. Арнольд В. И., Варченко А. Н., Гусейн-Заде С. М. Особенности дифференцируемых отображений. т. 1. Наука, М., 1982. 2. Арнольд В. И., Васильев В. А., Горюнов В. В., Ляико О. В. Особенности І. Локальная и глобальная теория. Итоги науки и техники. Соврем. проблемы математики. Фундаментальные направления, т. 6. 3. Danon J. A-equivalence and the equivalence of sections of images and discriminants. Lecture Notes in Math., Vol. 1462, 1989, pp. 93-121. 4. Mond D. Proc. London Math. Soc., 50, 333-369 (1985). 5. Shapiro B. Z. Math. Scand., 77, No. 1, 19-44 (1995). 